\title{
ERG expression in intraductal carcinoma of the prostate: comparison with adjacent invasive prostatic adenocarcinoma
}

\author{
Thomas M Schneider ${ }^{1}$ and Adeboye O Osunkoya ${ }^{1,2,3}$ \\ ${ }^{1}$ Department of Pathology, Emory University School of Medicine, Atlanta, GA, USA; ${ }^{2}$ Department of Urology, \\ Emory University School of Medicine, Atlanta, GA, USA and ${ }^{3}$ Emory Winship Cancer Institute, Atlanta, GA, \\ $U S A$
}

Intraductal carcinoma of the prostate is a growth pattern of prostatic adenocarcinoma that has not been well characterized from the molecular standpoint. It remains debatable whether intraductal carcinoma of the prostate represents colonization of benign glands by pre-existing conventional prostatic adenocarcinoma, or progression of high-grade prostatic intraepithelial neoplasia. TMPRSS2-ERG is the most common gene fusion in conventional prostatic adenocarcinoma, identified in about $40-70 \%$ of cases. In this study, we compared the expression of ERG in intraductal carcinoma of the prostate and adjacent conventional prostatic adenocarcinoma. Thirty-one confirmed cases of intraductal carcinoma of the prostate, with adjacent conventional prostatic adenocarcinoma and available tissue blocks, were identified at our institution. Immunohistochemical stains were performed for ERG using a rabbit anti-ERG monoclonal antibody. The ERG expression in the intraductal carcinoma of the prostate component was compared with that in the adjacent conventional prostatic adenocarcinoma. Mean patient age was 65 years (range: 48-79 years). Positive ERG expression was identified in $11 / 31(35 \%)$ cases of intraductal carcinoma of the prostate. In all $11 / 11(100 \%)$ cases with positive ERG expression in the intraductal carcinoma of the prostate component, ERG expression was also positive in the adjacent conventional prostatic adenocarcinoma. In the 20/31 cases with negative ERG expression in the intraductal carcinoma of the prostate component, ERG was also negative in the adjacent conventional prostatic adenocarcinoma. It is highly conceivable that based on the identical ERG expression (positive or negative) in intraductal carcinoma of the prostate and the adjacent conventional prostatic adenocarcinoma, intraductal carcinoma of the prostate most likely represents colonization of benign glands by adjacent pre-existing conventional prostatic adenocarcinoma.

Modern Pathology (2014) 27, 1174-1178; doi:10.1038/modpathol.2013.248; published online 10 January 2014

Keywords: ERG; high-grade prostatic intraepithelial neoplasia; intraductal carcinoma; prostate cancer; TMPRSS2-ERG

Intraductal carcinoma of the prostate is an uncommon growth pattern of prostate cancer. ${ }^{1}$ However, its recognition is important as intraductal carcinoma of the prostate has been shown in previous studies to be associated with high Gleason scores and tumor stage in radical prostatectomy specimens, and has an aggressive clinical course. ${ }^{2}$ Whether intraductal carcinoma of the prostate represents conversion of high-grade prostatic intraepithelial

Correspondence: Dr AO Osunkoya, MD, Department of Pathology Emory University School of Medicine Room H174 1364 Clifton Road, NE Atlanta, GA 30322, USA.

E-mail: adeboye.osunkoya@emory.edu

Received 31 October 2013; revised 5 December 2013; accepted 6

December 2013; published online 10 January 2014 neoplasia to carcinoma or invasion of benign glands by invasive prostatic carcinoma is still debatable. The transcriptional regulator $E R G$ is a member of the ETS gene family that can fuse with the TMPRSS2 protein. This gene has been found to be expressed in $40-70 \%$ of prostate carcinomas. ${ }^{1}$ Recent studies using FISH have shown that adjacent invasive carcinoma and intraductal carcinoma of the prostate share the same fusion gene status suggesting the two are clonally related. ${ }^{3}$ Only relatively few studies have analyzed the relationship of intraductal carcinoma of the prostate and invasive prostatic adenocarcinoma by immunohistochemistry. ${ }^{4}$ We sought to analyze the relationship between intraductal carcinoma of the prostate and adjacent conventional prostatic adenocarcinoma at 
our institution by using immunohistochemistry for ERG.

\section{Materials and methods}

A search was made through the surgical pathology and expert consult files of our institution for cases of intraductal carcinoma of the prostate from 2008 to 2012. Criteria proposed by Guo and Epstein ${ }^{5}$ was used to histologically diagnose specimens with intraductal carcinoma of the prostate. Their criteria defined intraductal carcinoma of the prostate as malignant epithelial cells filling large acini and prostatic ducts with preservation of basal cells and a solid or dense cribriform pattern or a loose cribriform or micropapillary pattern with either marked nuclear atypia with a nuclear size six times the normal or nonfocal comedonecrosis. ${ }^{5}$ Immunohistochemical stain for p63 was used to confirm retention of the basal layer in cases histologically consistent with intraductal carcinoma of the prostate. After the diagnosis of intraductal carcinoma of the prostate was confirmed, an immunohistochemical stain for the ERG protein was performed on all cases as follows: formalinfixed, paraffin-embedded prostate tissue from the various specimens was sectioned at $4 \mu \mathrm{m}$. Antigen retrieval was performed in Tris/borate/EDTA buffer, $\mathrm{pH} 8.0-8.5$, for $8 \mathrm{~min}$ at $95^{\circ} \mathrm{C}, 12 \mathrm{~min}$ at $100{ }^{\circ} \mathrm{C}$ and then $8 \mathrm{~min}$ at room temperature. Sections were then incubated with an anti-ERG rabbit monoclonal antibody (1:100 dilution, clone ID EPR3864, Epitomics, San Diego, CA, USA) for $32 \mathrm{~min}$ at room temperature. Subsequently, sections were incubated with a secondary antibody for $16 \mathrm{~min}$ at room temperature. The chromogenic substrate was applied for $8 \mathrm{~min}$ at room temperature. Slides were counterstained with hematoxylin. For the interpretation of ERG staining, only immunohistochemical stains with ERG-positive adjacent endothelial cells (internal control) were considered valid and only $2+/ 3+$ nuclear staining was considered positive. The expression of ERG in the adjacent conventional prostatic adenocarcinoma was also documented.

This study was completed following the guidelines of, and with approval from, our institutional review board.

\section{Results}

Mean patient age was 65 years (range: 48-79 years). The tissue specimens were as follows: 15/31 (48\%) cases from needle core biopsies (Figures 1a and b), $6 / 31(19 \%)$ cases from transurethral resections, $8 / 31$ $(26 \%)$ cases from radical prostatectomy specimens (Figures 1c and d), and 2/31 (7\%) cases from cystoprostatectomy specimens. Seven of 31 patients $(23 \%)$ had previously received androgen deprivation therapy for prior acinar prostatic adenocarcinoma, however, the areas with intraductal carcinoma of the prostate and adjacent acinar prostatic adenocarcinoma did not show therapy-related changes.

Positive ERG expression was identified in 11/31 $(35 \%)$ cases of intraductal carcinoma of the prostate (Figures 2a-c). In all 11/11 (100\%) cases with positive ERG expression in the intraductal carcinoma of the prostate component, ERG expression was also positive in the adjacent conventional prostatic adenocarcinoma (Figures 2b-d). In 20/31 (65\%) cases, there was negative ERG expression in the intraductal carcinoma of the prostate component and adjacent conventional prostatic adenocarcinoma (Figures 2e and f).

\section{Discussion}

Although the term intraductal carcinoma of the prostate was coined 40 years ago, we are just beginning to have a better understanding of the morphologic, immunohistochemical and molecular characteristics of this unique growth pattern of prostate cancer. ${ }^{1-6}$ There has been some interest over the years regarding the possible association between high-grade prostatic intraepithelial neoplasia and intraductal carcinoma of the prostate. The median risk of prostate cancer following a diagnosis of highgrade prostatic intraepithelial neoplasia is around $21 \% .{ }^{7}$ This risk is not significantly different from the risk of detecting prostate cancer following a benign diagnosis; and hence, the recommendations for urologists following diagnosis of high-grade prostatic intraepithelial neoplasia, especially if isolated, are controversial. Intraductal carcinoma of the prostate on the other hand, is strongly associated with a coexisting prostatic carcinoma. ${ }^{1,2,5,8,9}$ Therefore, the distinction between these two entities is of the upmost clinical significance.

In 2006, Guo and Epstein ${ }^{5}$ published their objective morphologic criteria for intraductal carcinoma of the prostate. It defined intraductal carcinoma of the prostate as malignant epithelial cells filling large acini and prostatic ducts with preservation of basal cells and either (1) a solid or dense cribriform pattern or (2) loose cribriform or micropapillary pattern with either marked nuclear atypia with a nuclear size six times normal or larger or nonfocal comedonecrosis. A dense cribriform pattern was defined where there were more solid and luminal areas, and nonfocal necrosis was considered when more than one duct showing comedonecrosis was seen. Using these criteria, they found similarly that intraductal carcinoma of the prostate is frequently associated with high-grade cancer and a poor prognosis. In their study, however, they only looked at cases in which no invasive carcinoma was identified in any of the cores. ${ }^{5}$ Other studies have shown that there is a spectrum of intraductal carcinoma of the prostate that does not fit into these 

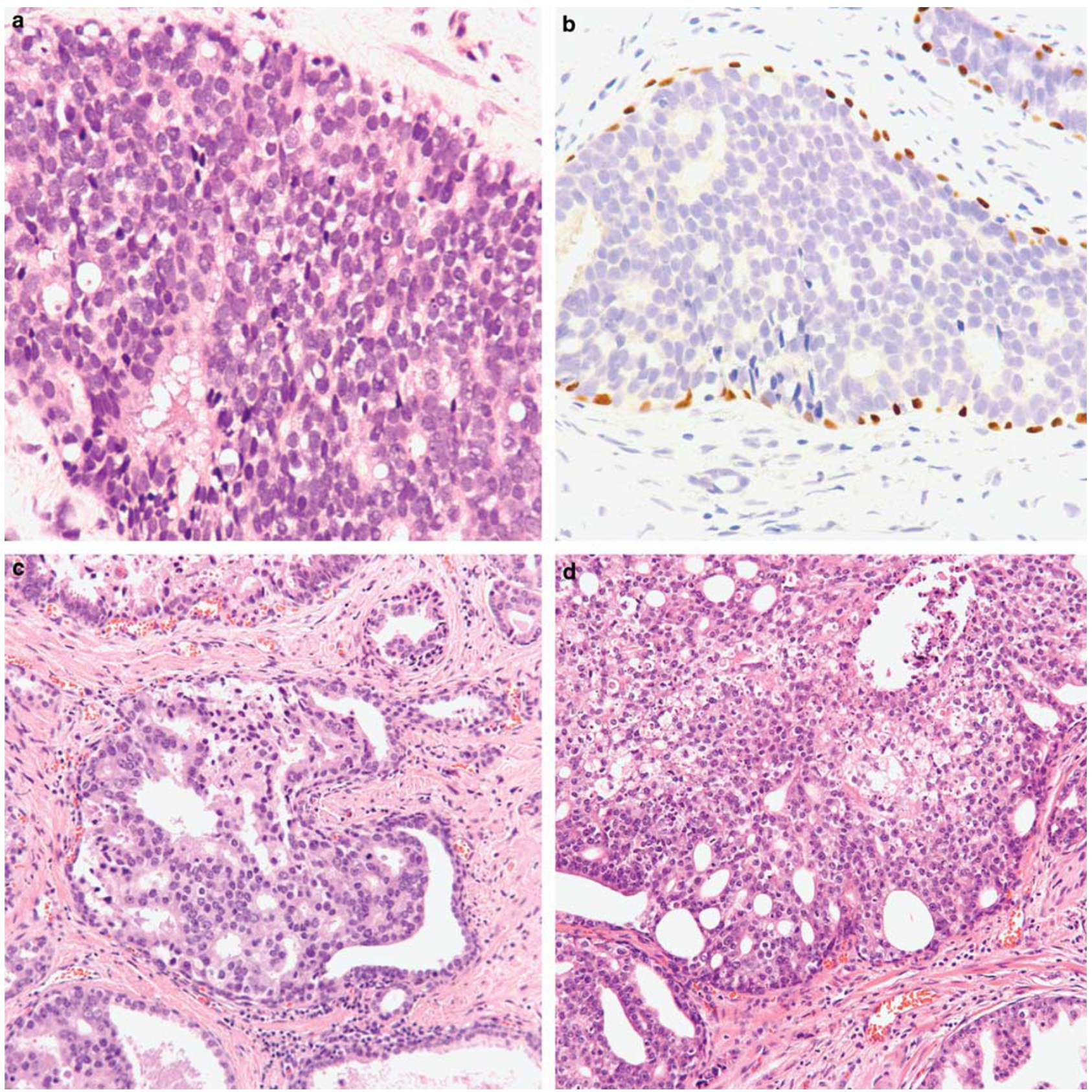

Figure 1 (a) Intraductal carcinoma of the prostate. Note the dense cribriform pattern and the marked nuclear atypia (hematoxylin and eosin). (b) Intraductal carcinoma of the prostate (corresponding to Figure 1a, p63 demonstrating retention of the basal cells). (c) Intraductal carcinoma of the prostate. Note that basal cells are apparent, and the tumor appears to be colonizing a previously benign gland (hematoxylin and eosin). (d) Intraductal carcinoma of the prostate with focal necrosis. Note that basal cells are apparent (hematoxylin and eosin).

criteria. A review of atypical cribriform lesions of the prostate by Shah and Zhou ${ }^{10}$ demonstrated that some cases of 'low-grade' intraductal carcinoma of the prostate, though distinct from cribriform highgrade prostatic intraepithelial neoplasia, may have some overlapping features.

In 2005, Tomlins et $a 1^{11}$ discovered chromosomal rearrangements in prostate cancer that fuse the androgen regulated gene transmembrane protease, serine 2 (TMPRSS2), with v-ets erythroblastosis virus E26 oncogene homolog $(E R G)$ of the ETS transcription factor family. Fusions of TMPRSS2 may occur with different members of the ETS transcription factor family, but $E R G$ was found to represent $\sim 90 \%$ of all ETS gene fusions. ${ }^{12}$ Later studies showed that $\sim 50 \%$ of prostate adenocarcinoma contains this gene fusion with significantly low staining $(\sim 1 \%)$ of benign prostatic tissue and 

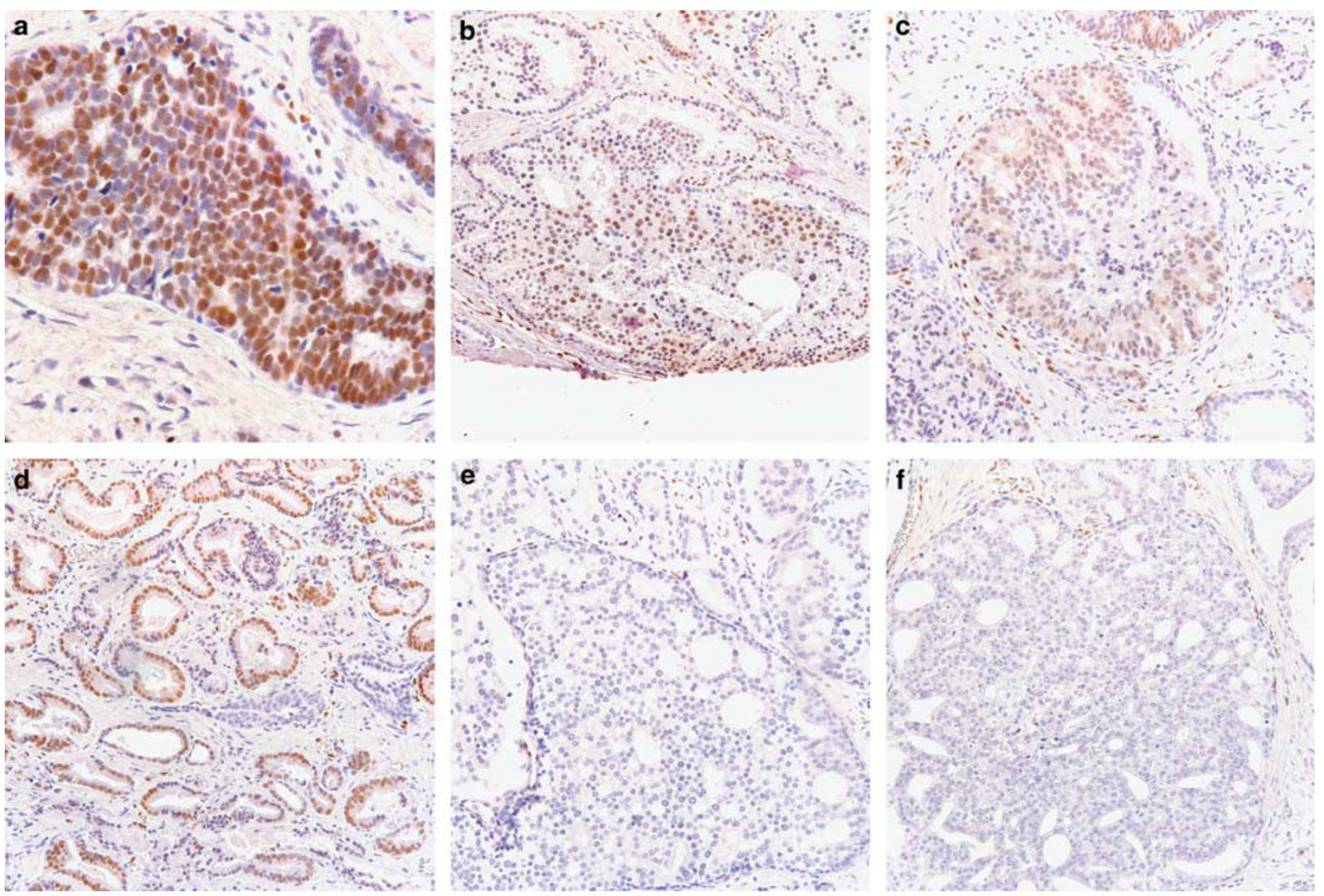

Figure 2 (a) Positive ERG expression in intraductal carcinoma of the prostate (corresponding to Figure 1a). (b and c) Positive ERG expression in intraductal carcinoma of the prostate and adjacent conventional prostatic adenocarcinoma. (d) Positive ERG expression in adjacent conventional prostatic adenocarcinoma. (e and f) Negative ERG expression in intraductal carcinoma of the prostate and adjacent conventional prostatic adenocarcinoma. Note the positive internal control (endothelial cells).

$18 \%$ of high-grade prostatic intraepithelial neoplasia. ${ }^{13}$ The detection of ERG in some cases of high-grade prostatic intraepithelial neoplasia, also lead to the investigation of ERG protein expression in prostate biopsies containing only high-grade prostatic intraepithelial neoplasia, and its possible utility in predicting subsequent cancer risks. A study by $\mathrm{He}$ et $a l^{14}$ found that ERG expression is distinctly uncommon (5.3\%) in cases of isolated high-grade prostatic intraepithelial neoplasia, and that positive ERG expression was not associated with increased cancer detection in subsequent repeat biopsies. The utility of the ERG antibody in the prediction of prostate cancer risk is therefore of limited value, and again emphasizes the importance of the distinction between intraductal carcinoma of the prostate and high-grade prostatic intraepithelial neoplasia on routine histologic examination. Lotan et $a l^{4}$ recently characterized the expression of ERG and PTEN in intraductal carcinoma of the prostate and high-grade prostatic intraepithelial neoplasia. PTEN had 92\% concordance between intraductal carcinoma of the prostate and ERG, and PTEN loss was never seen in high-grade prostatic intraepithelial neoplasia. They concluded that PTEN loss was a better marker to distinguish intraductal carcinoma of the prostate from high-grade prostatic intraepithelial neoplasia. ${ }^{4}$

$E R G$ rearrangements appear to be highly clonal because nearly all cells in a given cancer focus demonstrate positivity. ${ }^{13}$ It has also been recently shown that ERG is expressed in almost $50 \%$ of cases of mucinous prostatic adenocarcinoma and prostatic adenocarcinoma with mucinous features, similar to rates of expression in adjacent conventional prostatic adenocarcinoma, suggesting that these rare subtypes of prostatic adenocarcinoma are clonally related to conventional prostatic adenocarcinoma. ${ }^{15}$

All these factors make ERG an ideal marker for the comparison of expression between intraductal carcinoma of the prostate and adjacent conventional prostatic adenocarcinoma, in an effort to confirm its likely origin from the latter, as opposed to highgrade prostatic intraepithelial neoplasia. One would expect that if intraductal carcinoma of the prostate originated from the adjacent conventional prostatic carcinoma it would likely be from the same clone, and hence would have similar ERG expression. This was the finding in our study. On the whole $100 \%$ of intraductal carcinoma of the prostate cases staining 
for ERG stained positive in the invasive component, which highly suggests a clonal relationship of intraductal carcinoma of the prostate and adjacent conventional prostatic adenocarcinoma. The decreased frequency of ERG expression in this cohort, however, raises the possibility that the adjacent conventional prostatic adenocarcinoma in cases of intraductal carcinoma of the prostate, may have a slightly different pathobiology than the conventional prostatic adenocarcinoma not associated with intraductal carcinoma of the prostate.

This study emphasizes the fact that there is strong empirical evidence for intraductal carcinoma of the prostate being the result of the spread of existing carcinoma into benign ducts. Although there are rare reports of intraductal carcinoma without invasive carcinoma in adequately sampled prostates, nearly all cases of intraductal carcinoma of the prostate coexist with invasive prostate carcinoma. This is unique from any other organ site for carcinoma (especially breast), where intraductal carcinoma (ductal carcinoma in situ) is quite common without invasive breast carcinoma.

In conclusion, we have shown in our study that intraductal carcinoma of the prostate has $100 \%$ concordance of ERG positivity with adjacent conventional prostatic adenocarcinoma. This demonstrates that intraductal carcinoma of the prostate is clonally related to the adjacent conventional prostatic carcinoma and most likely represents colonization of benign glands.

\section{Disclosure/conflict of interest}

The authors declare no conflict of interest.

\section{References}

1 Osunkoya AO. Update on prostate pathology. Pathology 2012;44:391-406.

2 McNeal JE, Yemoto CE. Spread of adenocarcinoma within prostatic ducts and acini. Morphologic and clinical correlations. Am J Surg Pathol 1996;20: 802-814.

3 Han B, Suleman K, Wang L, et al. ETS gene aberrations in atypical cribriform lesions of the prostate: implications for the distinction between intraductal carcinoma of the prostate and cribriform high-grade prostatic intraepithelial neoplasia. Am J Surg Pathol 2010;34: 478-485.

4 Lotan TL, Gumuskaya B, Rahimi H, et al. Cytoplasmic PTEN protein loss distinguishes intraductal carcinoma of the prostate from high-grade prostatic intraepithelial neoplasia. Modern pathol 2013;26:587-603.

5 Guo CC, Epstein JI. Intraductal carcinoma of the prostate on needle biopsy: histologic features and clinical significance. Modern pathol 2006;19: 1528-1535.

6 Rhamy RK, Buchanan RD, Spalding MJ. Intraductal carcinoma of the prostate gland. J Urol 1973;109: 457-460.

7 Shepherd D, Keetch DW, Humphrey PA, et al. Repeat biopsy strategy in men with isolated prostatic intraepithelial neoplasia on prostate needle biopsy. J Urol 1996;156:460-462.

8 Pacelli A, Bostwick DG. Clinical significance of highgrade prostatic intraepithelial neoplasia in transurethral resection specimens. Urology 1997;50:355-359.

9 Henry PC, Evans AJ. Intraductal carcinoma of the prostate: a distinct histopathological entity with important prognostic implications. J Clin Pathol 2009; 62:579-583.

10 Shah RB, Zhou M. Atypical cribriform lesions of the prostate: clinical significance, differential diagnosis and current concept of intraductal carcinoma of the prostate. Adv Anat Pathol 2012;19:270-278.

11 Tomlins SA, Rhodes DR, Perner S, et al. Recurrent fusion of TMPRSS2 and ETS transcription factor genes in prostate cancer. Science 2005;310:644-648.

12 Tomlins SA, Bjartell A, Chinnaiyan AM, et al. ETS gene fusions in prostate cancer: from discovery to daily clinical practice. Eur Urol 2009;56:275-286.

13 Tomlins SA, Palanisamy N, Siddiqui J, et al. Antibodybased detection of ERG rearrangements in prostate core biopsies, including diagnostically challenging cases: ERG staining in prostate core biopsies. Arch Pathol Lab Med 2012;136:935-946.

14 He H, Osunkoya AO, Carver P, et al. Expression of ERG protein, a prostate cancer specific marker, in high grade prostatic intraepithelial neoplasia (HGPIN): lack of utility to stratify cancer risks associated with HGPIN. BJU Int 2012;110:751-755.

15 Johnson H, Zhou M, Osunkoya AO. ERG expression in mucinous prostatic adenocarcinoma and prostatic adenocarcinoma with mucinous features: comparison with conventional prostatic adenocarcinoma. Hum Pathol 2013;44:2241-2246. 